\title{
Design, Development and Evaluation of a Highly Versatile Robot Platform for Minimally Invasive Single-Port Surgery
}

\author{
Salman Can, Christoph Staub, Alois Knoll \\ Institute of Robotics and Embedded Systems \\ Technische Universität München \\ D-85748 Garching bei München, Germany \\ cans |staub|knollein.tum.de
}

\begin{abstract}
This work presents a new robot platform developed for laparoscopic single-port surgery. As a new approach, this platform enables the introduction of flexible endoscopic instruments through the designed hollow manipulators with $\emptyset 12 \mathrm{~mm}$ and 6 DOFs. Two such highly versatile manipulators and a 5 DOF telescope are combined in a unit that can be inserted through a $30 \mathrm{~mm}$ incision into the abdominal cavity. The entire platform is actuated by bowden wires in $2 \mathrm{~m}$ distance at the periphery. This design overcomes the problem of having a bulky and heavy platform near the patient. The design of the platform and the individual components are presented in this paper. The implemented control and simulation environment are also illustrated. The working range of one manipulator and the applicable forces were measured. Furthermore, the results of the accomplished in-vivo studies are presented.
\end{abstract}

\section{INTRODUCTION}

Laparoscopic surgery has many advantages such as trauma reduction, less pain, shorter hospitalization and better cosmetic results for the patient. However, a widespread application is still not possible due to limited flexibility, restricted visual access and poor ergonomics. Although considerable progress has been achieved with the introduction of computer assisted technologies and robotics, the intended breakthrough has not been reached. Further research is required to overcome the existing barriers such as limited flexibility, bulky design and high costs. Physicians are trying to develop new surgical procedures and techniques that could reduce trauma to the patient without compromising the surgical ability [1]. There are two new trends of surgical approaches, which are promising due to enhanced technical possibilities:

- Laparoscopic single-port surgery is performed through a single incision in the abdominal wall, where all the required instruments, usually up to four, are inserted.

- NOTES (Natural Orifice Transluminal Endoscopic Surgery) surgeries are performed through natural orifices and avoid an abdominal incision.

A consensus paper was already published, defining the challenges of single-port surgery [2]. In this regard the operational platform plays a central role for the progress of such demanding interventions. The goal is to have several independently controllable instruments and manipulators bundled together and introduced through a single access.

Laparoscopic single-port surgeries are currently performed using manually articulated instruments (e.g. RealHand, Autonomy Laparoangle) that are introduced through trocar-like

\author{
Adam Fiolka, Armin Schneider, Hubertus Feussner \\ Research Group MITI, Surgical Clinic \\ Klinikum rechts der Isar der TUM \\ D-81675 München, Germany \\ fiolka|schneider|feussner@mitigroup.de
}

devices (e.g. TriPort, SILS, Uni-X) with three channels [3]. TransEnterix has developed the SPIDER, a passive, sterile and disposable single-port platform, that achieves a better triangulation than rigid laparoscopic instruments [4]. Kaouk et al. reported the first series of single-port robotic procedures on humans using the daVinci system [5]. In addition to the development of new instruments for single-port interventions [6], Intuitive Surgical is also developing a new singleport manipulator claimed in a patent [7]. The crowding of instruments, triangulation, visualization, retraction and port related problems are limitations of the daVinci system that have to be overcome [8]. A new design proposed for singleport access surgery is the IREP [9]. This platform design with $17 \mathrm{DOF}$ in total comprises two snake-like continuum robots and a stereo vision module introduced through a $15 \mathrm{~mm}$ incision. Another robot platform proposed for singleport surgery is the SPRINT [10]. This platform with two 6 DOF robots, each with a diameter of $18 \mathrm{~mm}$ and integrated motors, is introduced through a $30 \mathrm{~mm}$ incision.

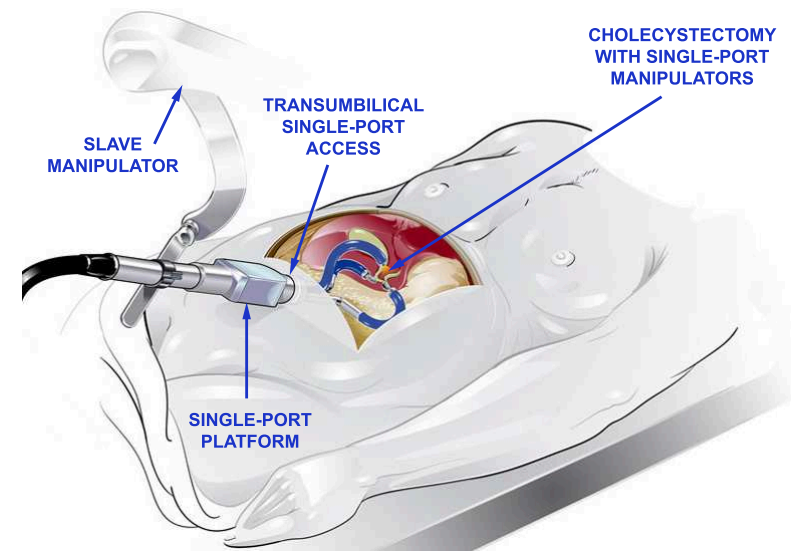

Fig. 1. Vision of the "single-port" platform: The highly versatile singleport system with two manipulators and a double-bending telescope that are inserted through a single incision into the abdominal cavity.

To overcome the existing challenges of single-port surgery, we developed the novel Highly Versatile Single-Port System (HVSPS). The concept of this platform is presented schematically in Figure 1 and 2. As a new approach, this platform comprises two hollow manipulators with $\emptyset 12 \mathrm{~mm}$ that provide the introduction of flexible endoscopic instruments up to $\emptyset 4 \mathrm{~mm}$. A double-bending telescope with $\emptyset 10 \mathrm{~mm}$ permits 
an enhanced visualization of the instruments in the large workspace of the entire system. Another important contribution of the developed system is the displacement of all the motor drives to the periphery, enabling the creation of a compact and lightweight platform deployed near the patient.

\section{Design Specifications And Considerations}

The requirements of the single-port platform were determined after evaluating several successful clinical experiments in close cooperation with the surgical team.

\section{A. Design Specifications}

The core concept behind the HVSPS system architecture consists of the following four components:

- Instruments: Multifunctional and flexible end-effectors introduced passively though the manipulators into the body. A tool changer charged with several instruments such as a grasper and scissors at the periphery permits the exchange of the instruments within seconds.

- Manipulators: Miniaturized and precisely controllable robots, integrated into a long and flexible carrier system with a highly flexible mechanism. The kinematic of the manipulator should enable intuitive manipulations comparable to a human arm i.e. a minimum of 6 DOFs.

- Telescope: A flexible double-bending endoscope providing the visualization of the surgical field and both manipulators in the required workspace in the abdomen.

- Carrier system: Comprises two manipulators for positioning the instruments and a highly flexible telescope. The carrier system provides, as a unit, the independent control of the manipulators and the telescope.

Cholecystectomy, a standardized laparoscopic operation, was chosen as the criterion for the definition of the surgical requirements. The necessary workspace of the instruments was defined according to an average gallbladder size with: $\mathrm{X} / \mathrm{Y}: \pm 50 \mathrm{~mm}$ and $\mathrm{Z}: \pm 30 \mathrm{~mm}$. This is larger than the workspace of the other proposed platforms. Depending on the surgical task, a force of at least $2 \mathrm{~N}$ must be provided at the instrument tip to achieve tissue manipulation [11]. From the surgical perspective the system should be able to operate at speeds of at least $50 \mathrm{~mm} / \mathrm{s}$ and have an accuracy exceeding $\pm 0.5 \mathrm{~mm}$. The final outer diameter of the inserted platform should be less than $22 \mathrm{~mm}$ (for the complete flexible system that can also be applied for NOTES procedures).

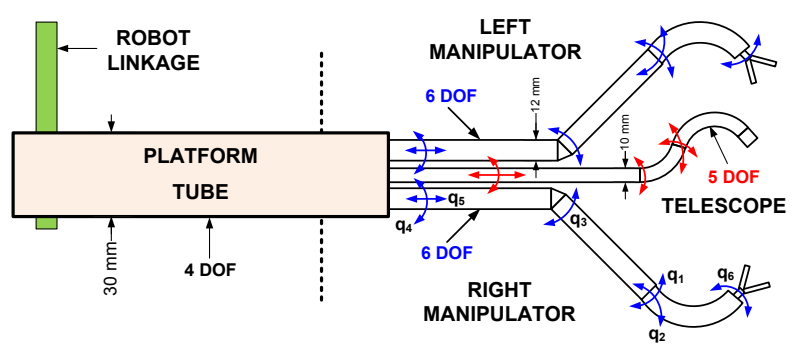

Fig. 2. Schematic drawing of the semi-rigid surgical single-port platform. Two 6 DOF manipulators $(\emptyset 12 \mathrm{~mm})$, a 5 DOF flexible telescope $(\emptyset 10 \mathrm{~mm})$ and a 4 DOF robot guiding the platform $(\emptyset 30 \mathrm{~mm})$.
TABLE I

SPECIFICATION OF THE OPERATING RANGE OF THE JOINTS

\begin{tabular}{|c|c|c|c|c|c|c|}
\hline & $\mathbf{q}_{1}$ & $\mathbf{q}_{2}$ & $\mathbf{q}_{3}$ & $\mathbf{q}_{4}$ & $\mathbf{q 5}$ & $\mathbf{q 6}$ \\
\hline Workspace & $\pm 180^{\circ}$ & $\pm 150^{\circ}$ & $\pm 90^{\circ}$ & $\pm 270^{\circ}$ & $80 \mathrm{~mm}$ & $\pm 90^{\circ}$ \\
\hline
\end{tabular}

\section{B. Design Considerations}

We intend to use electromagnetic position sensors for tracking the instruments. Furthermore, the surgeons desire to use the single-port platform during imaging procedures such as MRI or CT. In order to fulfill these requirements the drives are placed at the periphery in a distance of $2 \mathrm{~m}$. Cable driven mechanisms, flexible shafts, hydraulic or pneumatic mechanisms are possible solutions that can be used for the motion transmission. In this study, bowden wires were used to drive the HVSPS joints over long distances.

The development of manipulators with a hollow structure is a prerequisite in order to satisfy the requirement of interchangeability of the flexible instruments. In addition, an intuitive manipulation of tissue demands an opposable configuration of the manipulators for the exertion of traction and counter-traction. To meet these requirements, a flexible, hollow endoscope bending section with a diameter of $10 \mathrm{~mm}$ was used as the distal bending part of the manipulators.

In a first prototype, the diameter of the platform was restricted to $30 \mathrm{~mm}$, which is slightly bigger than the proposed single-port trocars and will be downsized in further studies.

\section{SySTEM DESCRIPTION}

The design of the HVSPS platform is shown in Figure 3. The manipulators and the telescope combined in the platform tube are introduced gas-tightly in a straight configuration through a trocar into the abdominal cavity. The platform is guided by a hydraulic telemanipulator, which is attached to the linkage tube. The guiding manipulator, with 4 DOFs, enables the pivoting of the system at the fulcrum, a linear movement into the body and the rotation of the platform. Figure 4 shows the distal section of the developed manipulators with the joints deflected to their maximum operating range. The bendable section of the manipulator with $2 \mathrm{DOF}$ has a length of $75 \mathrm{~mm}$ followed by a $50 \mathrm{~mm}$ long tube and an additional "elbow" articulation. Two further DOFs at the proximal end of each manipulator provide a rotation of $\pm 270^{\circ}$ and a linear movement of $80 \mathrm{~mm}$ into the abdominal cavity. The workspace of the manipulator joints are presented in Table I. A 5 DOF double-bending telescope also enables an S-shape configuration to visualization the instruments

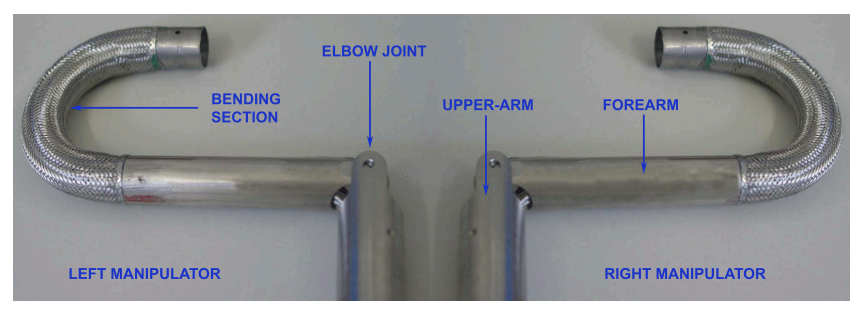

Fig. 4. Distal section of the HVSPS manipulators in opposed configuration. 


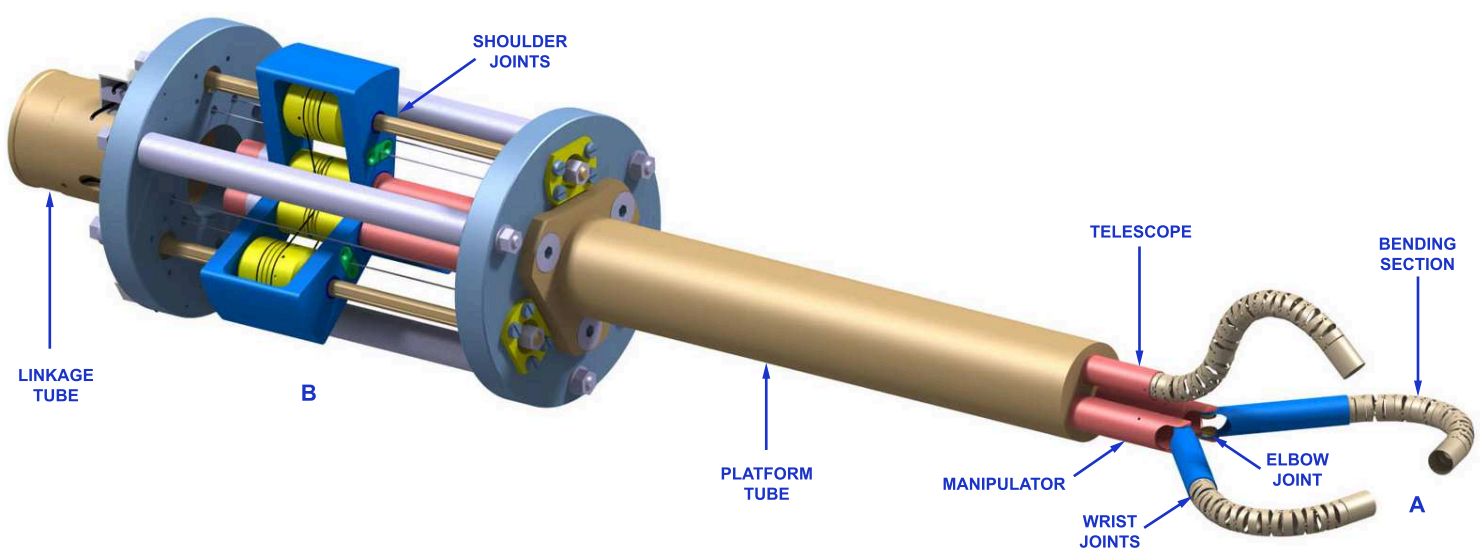

Fig. 3. Design of the "Highly Versatile Single-Port System". A: Distal articulations enabling a high dexterity inside the body. B: Actuation mechanism for rotation and linear motion of the manipulators and the telescope outside the body $(\emptyset 100 \times 160 \mathrm{~mm})$

in the entire workspace. The individual components of the single-port system are described subsequently.

\section{A. Bending Section}

The bending section of a flexible gastroscope with two DOFs is used as the distal bending section of the manipulators. It enables the introduction of the flexible instruments through its hollow structure. The bending section with $\emptyset 9 \mathrm{~mm} \times 75 \mathrm{~mm}$ consists of 20 parts. All of them are riveted together in a periodic arrangement and the distance between the hinges is $3.5 \mathrm{~mm}$. Figure 5 presents the configuration of the riveted joints. The bending section is mounted at the linkage part to the fore-arm tube and the deflection is realized by four bowden wires. Each of them is welded at the front part and guided through the lugs in the parts. The bending of the $13 \mathrm{Z}$-hinges by $\vartheta$ permits a deflection of more than $\pm 180^{\circ}$ in the horizontal plane and the bending of the $6 \mathrm{Y}$ hinges by $\phi$ a deflection of more than $\pm 150^{\circ}$ in the vertical plane. A deflection in each plane is realized by two bowden wires, i.e. the hinges are not actuated individually.

There are different ways to describe the kinematics of bending sections [12]. The bending section of an endoscope has a quasi-constant curvature. In order to describe the kinematics close to the physical system, we used the approach published by Lipkin et. al. [13]. For the determination of the instantaneous kinematics, we first treat the hinges as independent joints and then sum them up at the end, which results in a $2 \times 2$ Jacobian matrix. The kinematics for the integrated bending section with $n=19$ hinges is proposed subsequently.

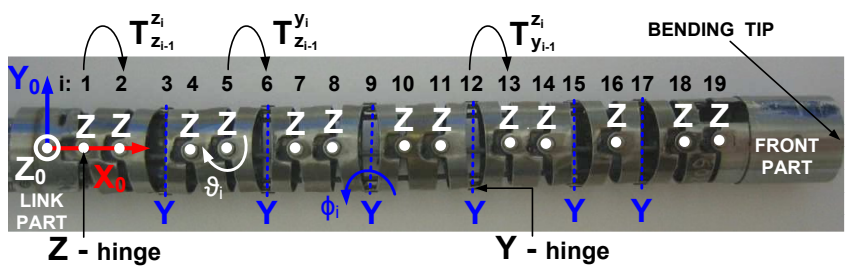

Fig. 5. Bending section of the flexible gastroscope illustrating the configuration of the hinges and their orientations.
The DH (Denavit-Hartenberg) parameters for $i=1 \ldots 19$ are: $a_{i}=3.5 \mathrm{~mm}, d_{i}=0$,

$$
\alpha_{i}=\left\{\begin{array}{lll}
0 & \text { for } & i=\{1,2,5,8,11,14,19\} \\
\pi / 2 & \text { for } & i=\{3,6,9,12,15,17\} \\
-\pi / 2 & \text { for } & i=\{4,7,10,13,16,18\}
\end{array}\right.
$$

Using these three cases results in the following three transformation matrices between the frames.

$$
T_{i-1}^{i}=\left\{\begin{array}{l}
T_{z_{i-1}}^{z_{i}}=\left[\begin{array}{cccc}
\cos (\vartheta) & -\sin (\vartheta) & 0 & 3.5 \\
\sin (\vartheta) & \cos (\vartheta) & 0 & 0 \\
0 & 0 & 1 & 0 \\
0 & 0 & 0 & 1
\end{array}\right] \quad \alpha_{i}=0 \\
T_{y_{i-1}}^{z_{i}}=\left[\begin{array}{cccc}
\cos (\phi) & -\sin (\phi) & 0 & 3.5 \\
0 & 0 & -1 & 0 \\
\sin (\phi) & \cos (\phi) & 0 & 0 \\
0 & 0 & 0 & 1
\end{array}\right] \quad \alpha_{i}=\frac{\pi}{2} \\
T_{z_{i-1}}^{y_{i}}=\left[\begin{array}{cccc}
\cos (\vartheta) & -\sin (\vartheta) & 0 & 3.5 \\
0 & 0 & 1 & 0 \\
-\sin (\vartheta) & -\cos (\vartheta) & 0 & 0 \\
0 & 0 & 0 & 1
\end{array}\right] \quad \alpha_{i}=-\frac{\pi}{2}
\end{array}\right.
$$

Consequently, the forward kinematics is calculated as follows

$$
T_{0}^{19}=T_{z_{0}}^{z_{1}} \cdot T_{z_{1}}^{z_{2}} \cdot T_{z_{2}}^{y_{3}} \cdot T_{y_{3}}^{z_{4}} \ldots \ldots T_{z_{16}}^{y_{17}} \cdot T_{y_{17}}^{z_{18}} \cdot T_{z_{18}}^{z_{19}}
$$

Twist velocity at the end frame relative to the base frame can be calculated using the $n \times 1$ array of hinge angle rates.

$$
t_{0}^{n}=\left[\begin{array}{c}
v_{0}^{n} \\
w_{0}^{n}
\end{array}\right]=J_{D} \dot{\theta} \quad \text { where } \quad \dot{\theta}=\left[\begin{array}{lllll}
\dot{\vartheta} & \dot{\vartheta} & \dot{\phi} & \ldots & \dot{\vartheta}
\end{array}\right]^{T}
$$

$J_{D}$ is the $6 \times n$ Jacobian matrix of the bending section.

$$
J_{D}=\left[\begin{array}{ccccc}
z_{0} \times p_{0}^{n} & \ldots & z_{i} \times p_{i}^{n} & \ldots & z_{n} \times p_{n}^{n} \\
z_{0} & \ldots & z_{i} & \ldots & z_{n}
\end{array}\right]
$$

Grouping all the partial velocities into the specific directions reduces the twist equation to:

$$
t_{0}^{n}=\dot{\vartheta} \cdot \sum_{i(z)}\left[\begin{array}{c}
z_{i} \times p_{i}^{n} \\
z_{i}
\end{array}\right]+\dot{\phi} \cdot \sum_{i(y)}\left[\begin{array}{c}
z_{i} \times p_{i}^{n} \\
z_{i}
\end{array}\right]
$$


For the calculation of the twist relative to the bending section tip, all the partial velocities are expressed in the end frame $n$. The vector $z_{i}$ in the frame $n$ can be expressed as follows:

$$
{ }_{n} \mathrm{Z}_{i}=T_{n}^{i} \cdot{ }_{i} \mathbf{Z}_{i}=\left(T_{i}^{n}\right)^{T} \cdot \cdot_{i} \mathbf{Z}_{i} \quad \text { with } \quad{ }_{i} \mathbf{Z}_{i}=\left[\begin{array}{lll}
0 & 0 & 1
\end{array}\right]^{T}
$$

The angular velocities of the axis $z_{n}$ and $y_{n}$ that are of interest to determine the two unknown angles $\vartheta$ and $\phi$ of the bending section, can be expressed in the end frame $n$ as:

$$
\left[\begin{array}{l}
\left({ }_{n} w_{0}^{n}\right)_{y} \\
\left({ }_{n} w_{0}^{n}\right)_{z}
\end{array}\right]=\left[\begin{array}{ll}
\sum_{i(z)}{ }_{n} z_{i} & \sum_{i(y)}{ }_{n} z_{i} \\
\sum_{i(z)}{ }_{n} z_{i} & \sum_{i(y)}{ }_{n} z_{i}
\end{array}\right] \cdot\left[\begin{array}{c}
\dot{\vartheta} \\
\dot{\phi}
\end{array}\right]
$$

Grouping all the velocity indices of both angles results a $2 \times 2$ matrix that can be easily inverted to calculate the velocity at the tool tip. These kinematics descriptions are also used to determine the overall kinematics of the manipulator.

\section{B. Elbow Joint}

The main challenges of the elbow joint are the hollow structure, which enables the introduction of flexible instruments through a working channel, and the need for a deflection of up to $\pm 90^{\circ}$ in order to achieve the intended workspace. To meet these requirements, we developed a new, bowden wire actuated joint as shown in Figure 6. A tiny pulley with $\emptyset 5 \times 1.5 \mathrm{~mm}$ is welded to each side in the forearm tube. The pivoting of the forearm tube is achieved by two studs that are screwed through the pulleys. The bowden wire sheaths are welded in the upper-arm tube and the $0.5 \mathrm{~mm}$ wires are wound one turn on the pulleys and welded to the forearm tube. The design of this elbow joint reduces the transmittable forces due to its low force translation ratio. The force reduction by the given ratio of the forearm tube plus bending section length and the pulley radius is: $i_{F}=125 \mathrm{~mm} / 2.5 \mathrm{~mm}=50$
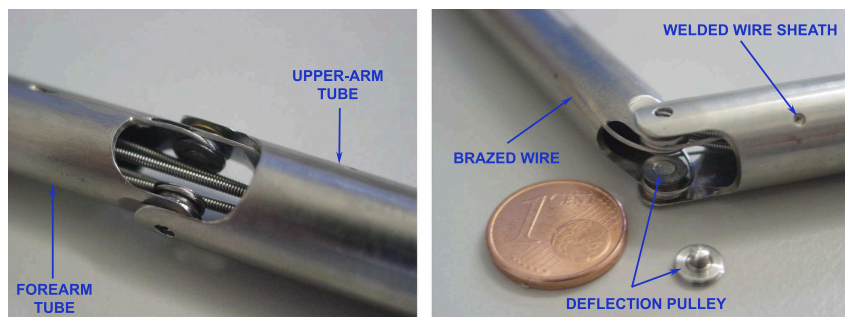

Fig. 6. Elbow articulation of the HVSPS manipulator.

\section{Rotary and Linear Drive Unit}

The rotation of the manipulator and the linear motion into the body are considered as shoulder articulations. Similar to the distal joints, all drives are placed at the periphery and the actuation of the passive mechanism is realized by bowden wires. The developed passive unit for this articulations, in total 6 DOFs for the two manipulators and the telescope, is shown in Figure 7. These articulations were implemented by using a THK LT6 ball spline. The ball spline pulley is integrated into an U-shaped "slider" and a bowden wire is attached on both sides of it. This enables a linear motion by pulling the wire to the respective side. One of the wires is deviated over a wheel on the base platform and both wire sheathes are attached to the top platform. The rotation of the ball spline is realized by a wire wound on the rotation pulley. Because of the small distance of less than $1 \mathrm{~mm}$ between the manipulators, the transmission of the rotation from the ball spline to the manipulator was achieved by a wire wound around the pulleys. Furthermore, floating bearings are integrated into the slider and the base platform to enable the guidance of the manipulators and the ball splines.

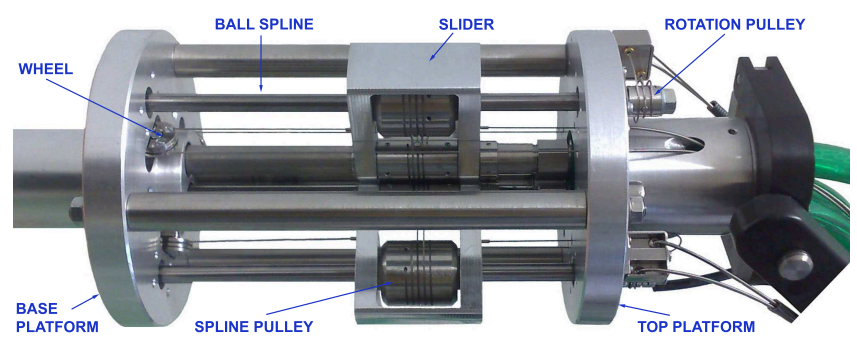

Fig. 7. Drive unit for rotation and linear actuation of the manipulators.

\section{Tool Changer}

Different from the other single-port systems, we are developing a new tool changer for the HVSPS that enables the exchange of flexible endoscopic instruments. The modular tool changer comprises a tool changing mechanism and four magazines. The linear motion guide of the changing mechanism enables switching between the different instruments. A controlled introduction and retraction of the appropriate instrument through the working channel of the manipulators is achieved by a clamping mechanism and an additional drive. Four different magazines with commercially available endoscopic instruments can be mounted on the changing mechanism. The handle of each instrument in the magazine is attached to a servomotor that opens or closes the instrument. Another important feature is the design of the tool changer with nonconducting materials to enable electrosurgery. The tool changer, i.e. the exchange of the instruments, is operated either by a hand-pad or by speech control.

\section{System CONTROL AND Simulation}

\section{A. Drive Unit and System Control}

The active drive unit of the platform is placed $2 \mathrm{~m}$ from the patient. The bowden wires of the manipulator joints are guided in a flexible hosing to the drive unit. In a gas-tight casing, the bowden wires are wound in pairs on pulleys and driven by DC motors. The tension of the wires can be adjusted by means of a screw coupling. The instrument channels of the manipulators, which are led through the active platform to the outside also offer the gas-tight connection to the tool changer and maintain thereby the aeroperitoneum. Each manipulator is connected as an individual module to the drive unit that permits an exchange in case of malfunction.

The overall system control is realized in two layers. Matlab-Simulink is used to implement the real-time, lowlevel control on one computer. The higher-level control with 
the calculation of the kinematics and path planning will be realized on a separate computer. A leaner Linux kernel with RTAI real-time extension is used for the control of the motor drives. Four motors are individually controlled over one Sensoray 626 I/O card. The encoder signals are fed directly over these cards to the computer, and a torque control is realized with PWM amplifiers. The implemented control provides a UDP interface for the higher-level control over the network. In its actual state, the joints of the HVSPS platform are controlled by using two joysticks and foot pedals.

\section{B. Simulation Environment of the Platform}

The simulation of the complete surgical scenario, with the HVSPS attached to the SoloAssist and mounted on an operating table, was implemented using the Coin3D open source library. The workspace of the manipulators, kinematic structure of the platform and motion modalities were evaluated and optimized using the simulation. For the training and teaching purposes of the physicians, a pick and place scenario was programmed, as shown in Figure 8. In this training exercise, surgeons had to grasp spheres with the instruments and place them at a predefined location. At the moment, the simulation works independent of the hardware. However, we plan to integrate them together to also enable an online visualization of the physical system.

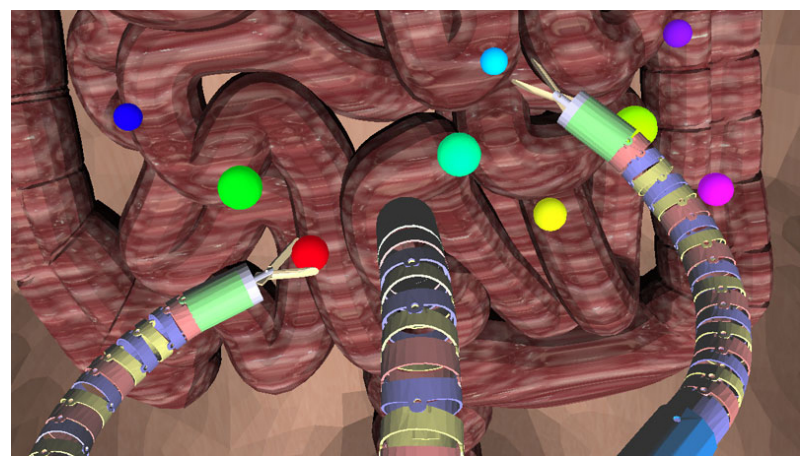

Fig. 8. Simulation environment of the HVSPS platform: Pick and place scenario for the purpose of training the physicians.

\section{EVALUATION}

\section{A. Determination of the Workspace}

First, the distal dexterity of the manipulator was investigated. For this task the Aurora (NDI) electromagnetic tracking system was deployed. Two 5 DOF sensors were used to determine the workspace of the manipulator tip. One of the sensors was mounted on the elbow joint which served as a reference and the second was introduced through the instrument channel and fixed at the tip. The position of the tip was then determined in relation to the elbow. Four series of measurements were carried out and the measurement with the largest deviation is described subsequently.

The theoretical workspace (red hatching in Figure 9) was determined based on the assumption of a length of $122 \mathrm{~mm}$ for the upper-arm tube including the bending section with a constant curvature that resembles a crescent shape with an inner radius of $51 \mathrm{~mm}$ and outer radius of $122 \mathrm{~mm}$. The thicker blue lines illustrate the spline-curve of the measured points. The maximum operating range is presented by the outer line and the inner curve presents the range of motion of the bending section. The large deviation of $11 \mathrm{~mm}$ at the top of the crescent can be justified by the inconstant movement of the bending section. This inaccuracy occurs mainly at a deflection of more than $90^{\circ}$. Apart from the large deviation at the crescent tip, the measured points, with a maximum deviation of $4.9 \mathrm{~mm}$, can be considered as fairly accurate. The average deviation to the border of the theoretically defined working range is less than $2.5 \mathrm{~mm}$. These inaccuracies should not be equated with the absolute positioning accuracy which can be improved by integrating sensor modalities.

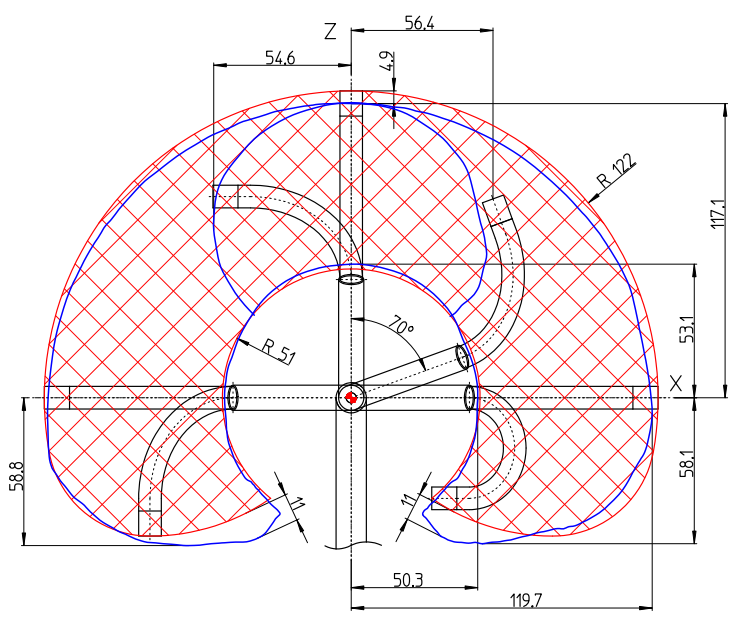

Fig. 9. Workspace evaluation of the distal dexterity: Red hatching presents the theoretical and the thicker blue line presents the measured workspace.

The other two DOFs of the manipulator expand the workspace so that the presented crescent is rotated $360^{\circ}$ about the $\mathrm{Z}$-axis and moved $80 \mathrm{~mm}$ in the $\mathrm{Z}$ direction into the body. At an angle of $70^{\circ}$, as shown in Figure 9, the horizontal distance of the instrument is $56.4 \mathrm{~mm}$ to the center of the manipulator and $63.8 \mathrm{~mm}$ to the center of the insert. Herewith it can be shown that the required workspace of $100 \times 100 \times 60 \mathrm{~mm}$ can be attained with the HVSPS platform.

\section{B. Evaluation of the Forces}

The force gauge FH500 (Sauter, Germany) was used to determine the achievable forces on the manipulator. The gauge was mounted horizontally on a test rig that can run a continuous push and pull motion. A series of 10 measurements were performed to determine the average force applicable by each joint. Measured forces are illustrated in Figure 10 with an error of approx. 0.1N.

A force of at least $1.8 \mathrm{~N}$ was achieved with the bending section in both directions which represents the wrist articulation. Measurements at the elbow were rather small in comparison due to the low transmission ratio of the joint. With the elbow motion, a force of $1.6 \mathrm{~N}$ could be applied at the wrist and $0.6 \mathrm{~N}$ on the tip. Furthermore, a force of more than $10 \mathrm{~N}$ was measured by the pulling and pushing of the 
manipulator with the linear joint. The rotation joint provides a torque of $0.17 \mathrm{Nm}$ that was measured at the wrist with an average force of $3.4 \mathrm{~N}$.

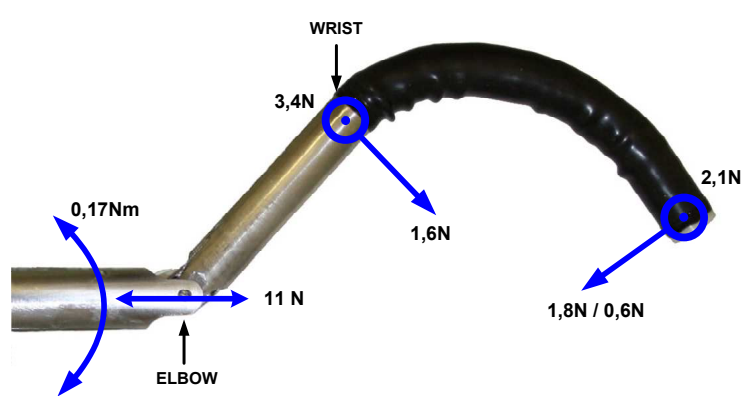

Fig. 10. Evaluation of the applicable forces on the HVSPS manipulator.

\section{Laparoscopic In-vivo Cholecystectomy}

First evaluations were carried out in 2008 for the early determination of the surgical requirements. Several invitro evaluations, performed on the human mock-up ELITE trainer, helped to improve the hardware before the successful realization of the first gallbladder dissection [14]. In the meantime, two further cholecystectomies were successfully performed with the HVSPS platform in animal studies (Figure 11). In these experiments, the set-up and operating time was reduced through the achieved enhancement of the hardware and accumulated experience. The operation time of these single-port laparoscopic surgeries ranged between 95130 minutes. The system was operated by two surgeons and an assistant. These experiments showed that the obtainable forces were sufficient to manipulate the gallbladder, however, it was not enough to hold the liver, for example, out of the operating field. The required movements were performed intuitively using the provided workspace.

\section{CONCLUSION AND OUTLOOK}

We presented the design and development of a novel platform for laparoscopic single-port surgery. The individual components of the system and parts of the kinematics have been described. The results of the workspace evaluation, the obtainable forces and clinical studies of the platform were presented. The feasibility of single-port operations were demonstrated in several experiments by using the developed system. With this study it was also shown that the desired objectives were largely met with the first prototype. It should be noted that bowden wires offer the advantage of controlling the joints from a distance, however, they introduce new challenges such as friction, backlash and low power transmission that have to be dealt with. This drawback can be partially mitigated by an adequate control that is adapted to the physical system. In a next step, the hardware will be optimized and reduced in size towards a fully flexible platform that also enables enhanced NOTES interventions. Furthermore, the Cartesian control of the tool tip, integration of an appropriate man-machine interface and sensor modalities will be implemented in the near future.

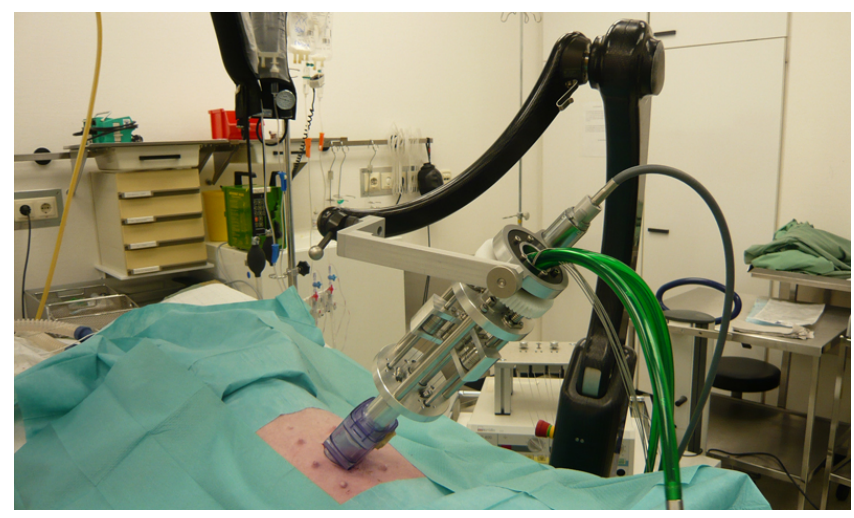

Fig. 11. In-vivo evaluation of the HVSPS in an animal study.

Further evaluations such as position accuracy, stiffness and friction will be analyzed after the integration of sensors.

\section{REFERENCES}

[1] H. Feussner, S. Can, A. Fiolka, and A. Schneider, "Hybrid surgery-the way towards notes the challenge for computer science," in Biomedical Imaging: From Nano to Macro, 2008. ISBI 2008. 5th IEEE International Symposium on. IEEE, 2008, pp. 1383-1386.

[2] I. Gill, A. Advincula, M. Aron, J. Caddedu, D. Canes, P. Curcillo, M. Desai, J. Evanko, T. Falcone, V. Fazio, et al., "Consensus statement of the consortium for laparoendoscopic single-site surgery," Surgical endoscopy, vol. 24, no. 4, pp. 762-768, 2010.

[3] J. Romanelli and D. Earle, "Single-port laparoscopic surgery: an overview," Surgical endoscopy, vol. 23, no. 7, pp. 1419-1427, 2009.

[4] A. Pryor, J. Tushar, and L. DiBernardo, "Single-port cholecystectomy with the TransEnterix SPIDER: simple and safe," Surgical endoscopy, vol. 24, no. 4, pp. 917-923, 2010.

[5] J. Kaouk, R. Goel, G. Haber, S. Crouzet, and R. Stein, "Robotic single-port transumbilical surgery in humans: initial report," $B J U$ international, vol. 103, no. 3, pp. 366-369, 2009.

[6] G. Haber, M. White, R. Autorino, P. Escobar, M. Kroh, S. Chalikonda, R. Khanna, S. Forest, B. Yang, et al., "Novel robotic da vinci instruments for laparoendoscopic single-site surgery," Urology, 2010.

[7] D. Larkin, T. Cooper, C. Mohr, and D. Rosa, "Minimally invasive surgical instrument advancement," June 13 2007, uS Patent App. $11 / 762,161$.

[8] G. Rao, M. Mansard, P. Ravula, P. Rebala, R. Dama, and D. Reddy, "Single-port surgery: Current applications and limitations," Asian Journal of Endoscopic Surgery, vol. 2, no. 3, pp. 56-64, 2009.

[9] K. Xu, R. Goldman, J. Ding, P. Allen, D. Fowler, and N. Simaan, "System design of an insertable robotic effector platform for single port access (SPA) surgery," in Intelligent Robots and Systems, 2009. IROS 2009. IEEE/RSJ International Conference on. IEEE, 2009, pp. $5546-5552$

[10] M. Piccigallo, U. Scarfogliero, C. Quaglia, G. Petroni, P. Valdastri, A. Menciassi, and P. Dario, "Design of a novel bimanual robotic system for single-port laparoscopy," Mechatronics, IEEE/ASME Transactions on, vol. 15 , no. 6 , pp. 871-878, 2010.

[11] J. Peirs, J. Clijnen, D. Reynaerts, H. Brussel, P. Herijgers, B. Corteville, and S. Boone, "A micro optical force sensor for force feedback during minimally invasive robotic surgery," Sensors and Actuators A: Physical, vol. 115, no. 2-3, pp. 447-455, 2004.

[12] R. Webster and B. Jones, "Design and kinematic modeling of constant curvature continuum robots: A review," The International Journal of Robotics Research, vol. 29, no. 13, p. 1661, 2010.

[13] H. Lipkin, J. Munnae, G. Zhou, and W. Daley, "Endoscope Kinematics," in 12th IFToMM World Congress, Besanon, June 18-21, 2007.

[14] S. Can, H. Mayer, A. Fiolka, A. Schneider, D. Wilhelm, H. Feussner, and A. Knoll, "The Highly Versatile Single Port System for laparoscopic surgery: Introduction and first clinical application," in 4th European Conference of the International Federation for Medical and Biological Engineering. Springer, 2009, pp. 1650-1654. 